\title{
ACESSO AO TRATAMENTO DO CÂNCER BUCAL NA REGIÃO DO COLEGIADO DE GESTÃO REGIONAL DE CAMPINAS - SP: ESTUDO DE CASO.
}

\author{
ACCESS TO ORAL CANCER TREATMENT IN THE REGIONAL \\ MANAGEMENT COLLEGIATE REGION ("COLEGIADO DE GESTÃO \\ REGIONAL - CGR") OF CAMPINAS, SP: A CASE STUDY
}

\author{
Elisa Toledo de Mesquita Sampaio* \\ Arlete Maria Gomes Oliveira" \\ Antônio Carlos Pereira*** \\ Marcelo de Castro Meneghim ${ }^{* * * *}$
}

\begin{abstract}
RESUMO
O objetivo desta pesquisa foi avaliar o desfecho dos casos com diagnóstico de câncer bucal nos serviços de referência em oncologia na região do Colegiado de Gestão Regional (CGR) de Campinas-SP. Estudo do tipo transversal com análise de dados secundários. Foram Avaliados 7 CEOs abrangendo 1.596.728 habitantes. Trata-se de pesquisa exploratória realizada em relatórios gerenciais do CEO Municipal e informações em Sistemas Regionais. Os dados foram coletados a partir de registros de pacientes dos serviços; demografia; tipo histológico, lesão local; diagnóstico de biópsia; data de encaminhamento e primeira consulta no centro de referência; início, conclusão e resultado do tratamento. Os resultados apontam que 94,7\% eram homens; $84,2 \%$ tinham idade $>50$ anos. O tempo entre detecção da lesão e confirmação do diagnóstico $<30$ (78,9\%) dias. Município de referência priorizado em 47,4\% dos casos. Os CEOs não tinham registro do tempo decorrido entre diagnóstico e início do tratamento para maioria dos casos, em que a situação de 52,6\% dos pacientes é desconhecida para CEOs, com exceção do município de Amparo. Conclui-se ser necessário um melhor acompanhamento de pacientes com câncer oral na região de Campinas (CGR).
\end{abstract}

Descritores: Câncer Bucal • Fatores de Risco • Integralidade em Saúde • Diagnóstico Precoce.

\section{ABSTRACT}

The objective of the research was to evaluate outcome of oral cancer cases diagnosed at oncology treatment reference services, Campinas Regional Management Collegiate, SP. Cross-sectional, observational epidemiological study conducted with secondary data analysis. Seven Dental Specialty Centers covering 1,596,728 inhabitants were evaluated in two stages. First, exploratory survey conducted of CEO's management reports, Municipal and Regional Information Systems. Secondly, data were collected from services' patient records: demographic; histological type, lesion site; biopsy diagnostic data; date of referral to, and first consultation at reference center; beginning and conclusion of treatment, outcome. Majority of cases were men (94.7\%); age $>50$ years $(84.2 \%)$. Mean time elapsed between lesion detection and diagnosis confirmation, $<30(78.9 \%)$ days. Municipality prioritized referral $(47.4 \%)$. CEOs had no record of time elapsed between diagnosis and initiation of treatment for most cases in which the situation of $52.6 \%$ of patients is unknown for CEOs, with the exception of Amparo municipality. We conclude that it is necessary a better follow up of oral cancer patients in Campinas, CGR region.

Descriptors: Mouth Neoplasms • Risk Factors • Integrality in Health • Early Diagnosis.

* Mestranda Odontologia (Saúde Coletiva) - Faculdade de Odontologia de Piracicaba - UNICAMP Univ. Estadual Campinas - SP. E-mail : elisatmsampaio@gmail.com

** Doutora em Odontologia (Saúde Coletiva) - Pós-Doutoranda - Faculdade de Odontologia de Piracicaba - UNICAMP Univ. Estadual Campinas - SP. E-mail: arlete.maria@yahoo.com.br

*** Livre Docente Odontologia - (Depart. Saúde Coletiva) - Professor Faculdade de Odontologia de Piracicaba - UNICAMP Univ. Estadual Campinas - SP. E-mail: apereira@fop.unicamp.br

**** Livre Docente Odontologia - (Depart. Saúde Coletiva) -Professor da Faculdade de Odontologia de Piracicaba - UNICAMP Univ. Estadual Campinas - SP. E-mail: meneghim@fop.unicamp.br 
I N T RO DUÇ Ã 0

As neoplasias malignas vêm se tornando um importante problema de saúde pública no Brasil e no mundo e, segundo a Organização Mundial de Saúde, a cada ano 10 milhões de casos novos são diagnosticados, transformando-se na segunda causa de morte no Brasil ${ }^{1,2}$

No ano de 2014 as taxas de incidência por 100 mil habitantes, no Brasil, estimaram um aumento de 15.290 novos casos de câncer bucal, representando o quinto tipo de câncer mais frequente em homens e o décimo segundo em mulheres ${ }^{3}$, e os determinantes socioeconômicos, em estudos mais recentes, vêm aparecendo como um fator com associação relevante ao aparecimento de novos casos de câncer da boca ${ }^{4}$.

O Brasil vem, desde 1998, estruturando sua política oncológica, estabelecendo requisitos para a garantia ao atendimento integral ao doente, parâmetros para o planejamento da assistência e definindo centros hospitalares que disponham de todos os recursos - CACON - Centro de Atendimento em Alta Complexidade em Oncologia, e UNACON - Unidade Assistencial de Alta Complexidade em Oncologia ${ }^{5}$. Os Centros de Especialidades Odontológicas (CEOs), são a principal estratégia de atenção secundária no Brasil para a detecção do câncer bucal ${ }^{6}$ oferecendo, entre suas especialidades, a área de Patologia para a detecção precoce.

O Projeto Onco Rede, iniciado em 2005, conta com a participação da Unicamp, gestores estaduais, municipais e equipes multiprofissionais dos municípios no entorno de Campinas, e tem como objetivo construir uma rede de cooperação entre os serviços municipais e os serviços especializados. O projeto é norteado por três eixos: acesso ao diagnóstico precoce do câncer e às ações de prevenção, gestão do cuidado e a qualificação da atenção nos municípios ${ }^{7}$. É importante relatar que a região de Campinas registrou, em 2013, 112 óbitos por neoplasia maligna do lábio, cavidade oral e faringe, e em 2012 ocorreram $144^{2}$.

Diante do exposto, faz-se necessária a pergunta: Como está a regulação e o cuidado dos pacientes diagnosticados com câncer bucal?

Para tanto, este estudo de caso tem o objetivo de compreender a realidade da região, de forma a contribuir para melhorar o sistema de saúde.

\section{MATERIAIS E MÉTODOS}

Trata-se de um estudo observacional transversal exploratório com análise secundária de dados, desenvolvido na região do Colegiado de Gestão Regional de Campinas. Em 2012, o Departamento Regional de Saúde 7 (DRS7) Campinas, da Secretaria de Estado da Saúde de São Paulo, estava dividido em 4 Colegiados - CGR Campinas, CGR Oeste, CGR Bragança Paulista e CGR Jundiaí, num total de 42 municípios. Foi selecionado o CGR Campinas, composto por 7 Centros de Especialidades odontológicas, com cobertura de 1.596.728 habitantes. No ano de 2011 foram analisados todos os casos de câncer bucal diagnosticados de forma positiva nessa região, num total de 26 indivíduos. A Regulação para os Serviços de Referência é feita pelo Núcleo de Regulação do DRS7, e estão habilitados os seguintes Serviços de Oncologia:

1. Centro Infantil de Investigação Hematológica Dr. Domingos A. Boldrini - Unacon, Exclusiva de Oncologia Pediátrica com Serviço de Radioterapia;

2. Hospital das Clínicas da UNICAMP - Cacon;

3. Hospital Municipal Dr. Mário Gatti - Unacon com Serviço de Radioterapia;

4. Hospital Celso Pierro / PUC Campinas - Unacon com Serviço de Hematologia.

Na primeira etapa, foi realizado um levantamento exploratório dos relatórios gerenciais dos CEOs, dos sistemas de informações dos municípios e região, com a finalidade de se identificar o tipo de CEO, a população atendida, o laboratório de referência, os serviços de referência para oncologia e o número de biópsias e de casos de câncer bucal confirmados. Em seguida, de posse dos dados confirmados sobre o número de biópsias, foi
SAMPAIO ETM OLIVEIRA AMG PEREIRA AC MENEGHIM MC

ACESSO AO TRATAMENTO DO CÂNCER BUCAL NA REGIÃO DO COLEGIADO DE GESTÃO REGIONAL DE CAMPINAS SP: ESTUDO DE CASO
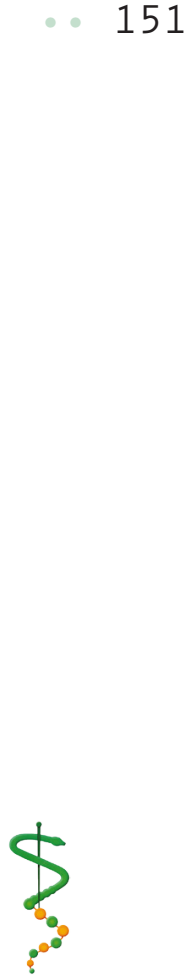

REV, ODONTOL, UNIV, CID , SÃO PAULO

2015; $27(2): 150-$ 55, MAI O-AGO 
SAMPAIO ETM

OLIVEIRA AMG

PEREIRA AC

MENEGHIM MC

ACESSO AO

TRATAMENTO DO

CÂNCER BUCAL

NA REGIÃO DO

COLEGIADO DE

GESTÃO REGIONAL

DE CAMPINAS -

SP: ESTUDO DE

CASO

152

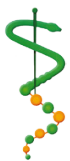

REV, ODONTOL.

UNIV, CID, SÃO

PAULO

$2015 ; 27(2): 150-$

55, MAIO-AGO
I SSN 1983-5183

realizada a revisão de prontuários dos pacientes identificados para levantamento de dados.

As variáveis independentes foram: gênero, idade, profissão, fatores de risco, tipo histológico da lesão, local da lesão, data da biópsia, data do diagnóstico, data do encaminhamento para referência e adesão ao tratamento do paciente. Nessa fase verificou-se junto aos prontuários o registro da primeira consulta em serviço de referência, data do início e término do tratamento e modalidade (cirurgia, radioterapia ou quimioterapia). No passo seguinte foram verificados o acompanhamento e o tratamento recebido, percentual de alta por cura, óbitos ou abandono de tratamento.

Foram excluídos do estudo os diagnósticos feitos por especialidades médicas e outros serviços da região do CGR Campinas, uma vez que os serviços especializados selecionados para o estudo, foram os Centros de Especialidades Odontológicas. Realizou-se análise descritiva dos dados por meio de tabela de frequência.

O presente estudo foi aprovado pelo Comitê de Ética em Pesquisa em Seres Humanos da FOP- Unicamp, conforme Normas do Ministério da Saúde, Resolução no 466 de 12 de dezembro de 2012, do Conselho Nacional de Saúde, Protocolo $n^{\circ}$ 018/2013, em 03 de julho de 2013.

\section{RESULTADOS}

Os dados foram obtidos a partir de levantamento das biópsias realizadas pelos CEOs da região do CGR Campinas. Do total de 431 biópsias, foram confirmados 26 casos de câncer bucal (6,0\% das biópsias). Destes, 7 foram excluídos da amostra por dificuldade de localização do prontuário, restando, portanto, 19 prontuários para serem analisados. A Tabela 1 apresenta a distribuição das variáveis sociais, clínicas e de organização do serviço em relação aos CEOs participantes da pesquisa.

Os resultados mostram que dos 19 pacientes diagnosticados com câncer bucal, $94,7 \%$ eram do gênero masculino, sendo que $15,8 \%$ tinham entre $20-49$ anos de idade, $36,8 \%$ entre $50-59$ anos e $47,4 \%$ acima de 60 anos. O tempo decorrido entre a detecção da lesão e o exame para diagnóstico nos CEOs foi, em média, inferior a 30 dias para 78,9\% dos usuários. A regulação dos encaminhamentos dos pacientes se deu de forma prioritária pelo município $(47,4 \%)$ e a DRS-7 $(21,1 \%)$. Ocorreram outras formas de regulação, mediadas pelo serviço privado e pelo CEO de Campinas que faz a regulação de forma direta com a Área de Cabeça e Pescoço da PUC-Campinas. Os CEOs, exceção ao de Amparo, não dispunham da informação quanto ao tempo decorrido entre a confirmação do diagnóstico da lesão até o início do tratamento. Ao se analisar a contra-referência, verificou-se que o destino dos pacientes é ignorado em 52,6 \% dos casos. Dos outros 9 pacientes, 7 foram a óbito $(36,8 \%), 1$ caso tem acompanhamento pelo CEO e 1 em serviço de oncologia.

\section{I SCUSSÃO}

De acordo com informações obtidas no Mapa de Saúde do Município de Campinas, Brasil*, a oferta de serviços de média complexidade constitui-se em um dos maiores pontos de estrangulamento do sistema, organizada e financiada historicamente com base na lógica da oferta de procedimentos, desconsiderando as necessidades e o perfil epidemiológico da população, configurando-se o município de Campinas como referência para a região no tratamento do câncer.

Os resultados encontrados neste estudo são similares aos de outros estudos realizados em outras regiões do Estado de São Paulo e do Brasil, com predominância de homens acima de 50 anos de idade, e com maior frequência de diagnóstico o carcinoma espinocelular 8,9

O tempo é essencial para o prognóstico desses pacientes, porém, neste estudo, não foi encontrado nenhum tipo de protocolo ou monitoramento dos casos de câncer bucal pelas unidades de saúde. Pôde-se observar que, em todos os municípios, as biópsias são realizadas com agilidade (menos de 30 dias para 78,9\% dos casos) e os resultados disponibilizados imediatamente para o serviço. Contudo, a dificuldade está no acesso aos 
Tabela 1 - Distribuição das variáveis associadas aos aspectos demográficos, acesso, referência e tratamento em relação ao local de diagnóstico .

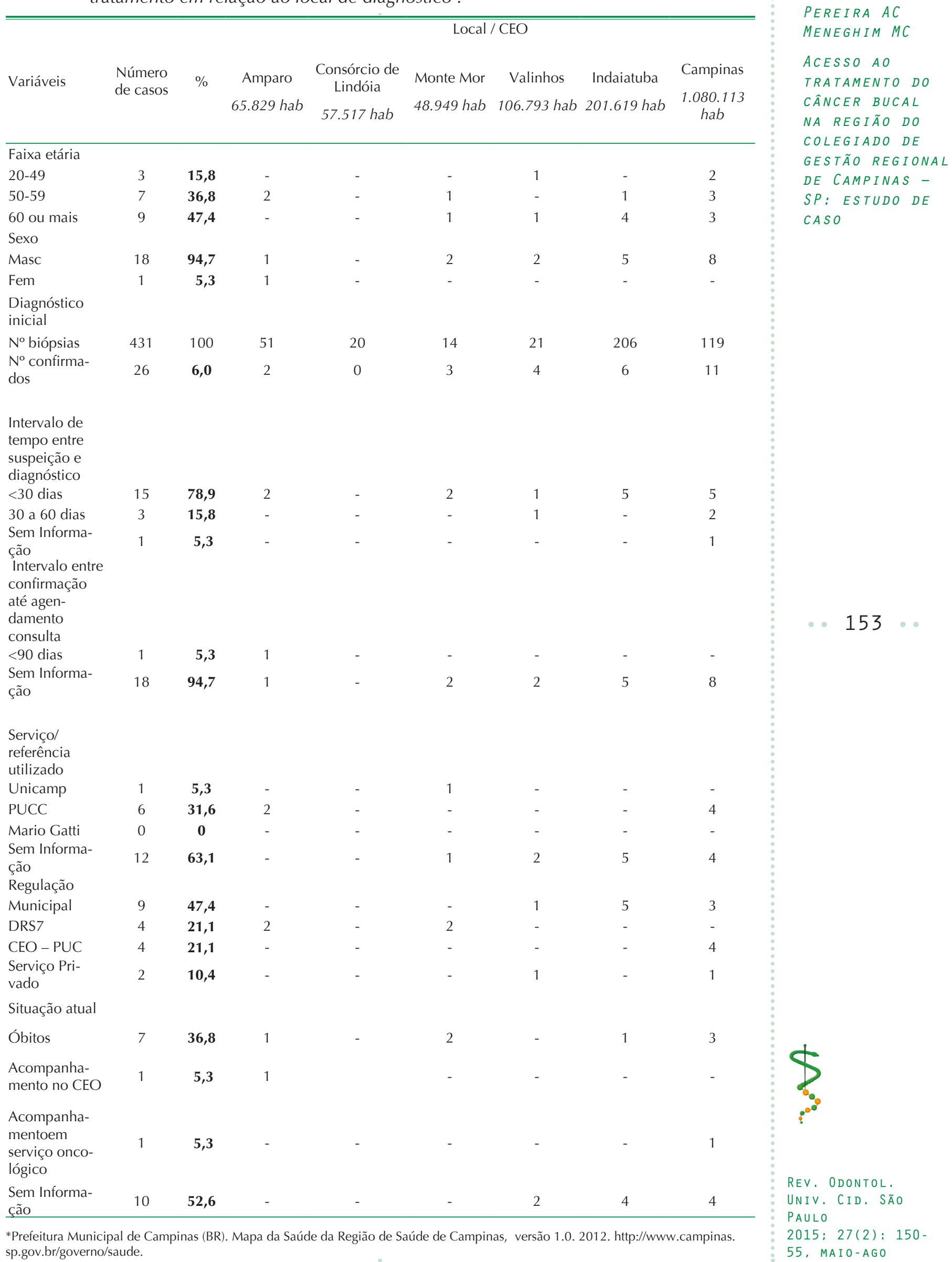


SAMPAIO ETM

OLIVEIRA AMG

PEREIRA AC

MENEGHIM MC

ACESSO AO

TRATAMENTO DO

CÂNCER BUCAL

NA REGIÃO DO

COLEGIADO DE

GESTÃO REGIONAL

DE CAMPINAS -

SP: ESTUDO DE

CASO

$154 \ldots$

REV, ODONTOL,

UNIV. CID, SÃO

PAULO

$2015 ; 27(2): 150-$

55, MAIO-AGO serviços de referência que, como destacado neste estudo, aproximadamente 95\% dos casos não apresentam informação relacionada.

Cabe ressaltar que o Brasil instituiu em 2012 a chamada "Lei dos 60 dias" a qual normatiza o prazo de 60 dias para o início do tratamento após o diagnóstico do câncer. Neste estudo, apenas o CEO do município de Amparo informou em cerca de 90 dias os casos diagnosticados. Outros estudos identificaram prazos maiores de 60 dias para o início do tratamento $^{10,11 .}$

Observou-se nos resultados desta pesquisa que a regulação do acesso dos casos de pacientes oncológicos é feita prioritariamente pela gestão municipal $(47,4 \%)$ e pelo Estado $21,1 \%$ ), como preconizado pelo SUS. No município de Campinas, além dos agendamentos regulados pelos serviços, também acontecem agendamentos fora da regulação municipal, por meio de contato direto com serviços de oncologia. Quanto ao cuidado com o paciente oncológico, verifica-se que o sistema de informação para essa rede de atenção está falho, pois, para 52,6\% dos casos, a situação é desconhecida. Assim, esses pacientes ficam perdidos entre a unidade de saúde que os encaminhou ao CEO, entre o CEO e a área de regulação municipal, e entre a regulação e o serviço de referência.

O trabalho em rede depende do estabelecimento de parcerias feitas com e entre profissionais dos municípios, como uma base forte e um ponto fundamental para que se configure uma rede capaz de atender as diferentes demandas dos pacientes, familiares e profissionais de saúde ${ }^{11}$.

Com a finalidade de mostrar algumas práticas, a partir da experiência com o le- vantamento dos dados, pode-se relatar o caso do CEO de Valinhos que propôs que um funcionário do CEO passe a acompanhar os casos através de telefonemas à família, para não perder contato e verificar o tempo de agendamento em serviço de referência; o município de Indaiatuba, que conta com médico oncologista na rede e cuida pessoalmente do encaminhamento aos serviços de referência para continuidade do tratamento (quimioterapia ou radioterapia), porém, os profissionais do CEO não são informados do destino e tratamento de cada um deles; e o CEO de Amparo que apresentava todas as informações através da profissional que atende à área e tem contato com o paciente e a família.

Os dados mostram uma contra-referência praticamente inexistente. O registro de informações encontrados nos prontuários é bastante reduzido e não há acompanhamento dos casos após diagnóstico e encaminhamento para serviço especializado. O número de óbitos $(36,8 \%)$ era desconhecido dos serviços e só foram verificados após a coleta de dados. Há a necessidade de melhor acompanhamento dos pacientes com câncer bucal na rede de atenção à saúde, visto que existe insuficiência de registros e, na maioria, os pacientes não vêm sendo monitorados pelos serviços de saúde da região de Campinas.

\section{CONCLUSÃO}

Conclui-se que há necessidade de melhor acompanhamento dos pacientes com câncer bucal na rede de atenção à saúde, visto que existe insuficiência de registros. A maioria dos pacientes atendidos pelos serviços de saúde da região de Campinas não vem sendo monitorada. 
1. Brasil. Leis e decretos. Lei $n^{0} 12.732$, de 22 de novembro de 2012. Dispõe sobre o primeiro tratamento de paciente com neoplasia maligna comprovada e estabelece prazo para seu início. Diário Oficial da União 2012a

2. Brasil.Secretaria de Estado da Saúde. Sistema de línformações de mortalidade. São Paulo 2013 [Acesso em 10 nov 2013]; Disponível em: http:// www.saude.sp.gov.br/ses/informacoes-de-saude-tabnet

3. Brasil. Ministério da Saúde. Estimativa 2014: incidência de câncer no Brasil. Rio de Janeiro: Instituto Nacional do Câncer José Alencar Gomes da Silva (INCA); 2014 [Acesso 2015 agosto 10]; Disponível em: http://www. inca.gov.br/estimativa/2014/estimativa-24042014.pdf

4. Torres-Pereira CC, Angelim-Dias A, Melo NS, Lemos Jr. CA, Oliveira EMF. Abordagem do câncer da boca: uma estratégia para os níveis primário e secundário de atenção em saúde. Cadernos de Saúde Pública 2012 28(suppl):s30-s9.

5. Brasil. Ministério da Saúde. Estimativas 2012: incidência de câncer no Brasil. Rio de Janeiro: INCA; 2012b [Acesso 2014 jul 24]; Disponível em: http://www.inca.gov.br.

6. Goes P, Moysés S. Planejamento, gestão e avaliação em saúde bucal. São Paulo: Artes Médicas; 2012.
7. Santiago S, Andrade G. A educação permanente como construtora do cuidado integral e do trabalho em rede na atenção a pacientes com câncer na região de Campinas/SP. Campinas: Unicamp/FCM; 2012.

8. Oliveira LR, Ribeiro-Silva A, Zucoloto $S$. Perfil da incidência e da sobrevida de pacientes com carcinoma epidermóide oral em uma população brasileira. Jornal Brasileiro de Patologia e Medicina Laboratorial 2006 out;42(5):385-92.

9. Simões Nogueira A, Alves Pereira KM, Turatti E, Cavalcante Pouchain E, Gurgel Costa FW, Albuquerque Taboza Z, et al. Perfil Epidemiológico de 23 casos de neoplasias malignas da cavidade oral atendidos em uma instituição odontológica de nível secundário. Rev Bras Cir Cabeça Pescoço 2012 out/ dez;41(4):

10. Costa E, Magliorati C. Câncer bucal: avaliação do tempo decorrente entre a detecção da lesão e o início do tratamento. Rev Bras Cancerol 2001 2839;47(3):

11. Sommerfeld CE, Santiago SM, Andrade MdGG, Peroni FMA. Impacto do trabalho integrado em rede no diagnóstico precoce do câncer bucal. Rev Bras Cir Cabeça Pescoço 2013 mar;2013(42):1.

Recebido em 31/11/2015

Aceito em 16/11/2015
SAMPAIO ETM OLIVEIRA AMG PEREIRA AC MENEGHIM MC

ACESSO AO TRATAMENTO DO CÂNCER BUCAL NA REGIÃO DO COLEGIADO DE GESTÃO REGIONAL DE CAMPINAS SP: ESTUDO DE CASO

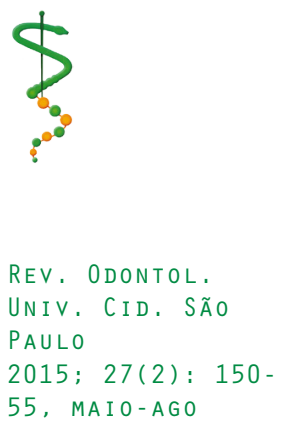

